\title{
Exosomes: Human Saliva-derived nanoBiomarkers for Use in Clinical Dentistry?
}

\author{
Exosomas: ¿nanoBiomarcadores Derivados de \\ Saliva Humana para Uso en Odontología Clínica?
}

EDITORIAL

Ziyad S. Haidar ${ }^{1,2,3,4}$

Exosomes are small (30-120 $\mathrm{nm})$ endocytic cell membrane-derived nano-vesicles (from the larger $\sim 1$ $\mathrm{mm}$ late endosomal multivesicular body-derived circulating vesicles; after fusion with the plasma membrane - released from activated cell surfaces), discovered in the mid-80s, and for long considered to serve as "garbage bags" for cells to discard undesirable cargo. Today, exosomes are attracting exquisite snow-balling attention as they are known to be present in all biological fluids including blood, urine, amniotic fluid, breast milk, ascitic and hydrothoracic fluid. This also includes non-invasively obtained bodily fluids such as saliva, harboring a variety of proteins, nucleic acids, and lipids (predominantly cholesterol, sphingolipids, phospholipids, and bisphosphates). Exosomes have a unique and complex composition. Indeed, the latest exosome content database identifies: 4,563 proteins, 194 lipids, 1,639 mRNAs, and 764 miRNAs, in exosomes from multiple organisms (Exocarta V4; available at: www.exocarta.org). Exosomes, which carry various molecular constituents of their cell of origin, including proteins, lipids, mRNAs, and microRNAs (miRNAs), have characteristic surface markers (such as CD63, CD9, CD81, and TSG101) and participate in cell-to-cell communications and protein and RNA delivery, thereby, influence physiological as well as pathological processes (note: exosomes are shed by cells under both normal and pathological conditions). For example, while several studies report that miRNA contents of circulating exosomes correspond to those of their originating cancer cells (tumor cells release exosomes containing tumor-specific RNAs), other studies report that miRNAs can be detected in exosomes isolated from saliva. Hence, exosomes are suggested to have potential for cancer diagnostics and offer a great utility promise as novel biomarkers (and perhaps, targeted delivery systems) for clinical diagnosis and management/ treatment of pathologies ad conditions. As mentioned earlier, while exosomes originate (or are released - via exocytosis) from many cell types (primarily, hematopoetic cells), such as platelets, reticulocytes, lymphocytes ( $\mathrm{B}$ and $\mathrm{T}$ ), mast cells, epithelial cells, endothelial cells, dendritic cells, macrophages and neurons; molecular constituents are often associated with certain diseases and treatment responses. Thus, exosomes have also emerged as an exciting potential candidate for immune-therapy, vaccination and as a novel vector for gene therapy, beyond diagnostics.

Given their small size and low density, exosomes are usually isolated from bodily fluids and cell culture media via labor-intensive, technically-sensitive, timeconsuming and expensive differential ultracentrifugation, in a laboratory setting, typically. To obtain exosomes from human saliva, for example, stimulated submandibular/sublingual and parotid glandular saliva (bi-lateral, using $2 \%$ citric acid; repeated up to 8-10 times) is collected and immediately placed on ice, and centrifuged at $1500 \mathrm{~g}$ for $10 \mathrm{~min}$ at $4{ }^{\circ} \mathrm{C}$. The supernatant is then removed, placed in another tube and centrifuged at $17000 \mathrm{~g}$ for $15 \mathrm{~min}$ at $4^{\circ} \mathrm{C}$ to further remove unwanted organelles and cell fragments. The supernatant is again transferred to sterile tubes and ultra-centrifuged at $160000 \mathrm{~g}$ for $1 \mathrm{~h}$ at $4^{\circ} \mathrm{C}$. The pellet containing the exosomes is washed with phosphatebuffered saline and ultra-centrifuged again at 160000 $\mathrm{g}$ for $1 \mathrm{~h}$ at $4{ }^{\circ} \mathrm{C}$. Again, the supernatant is removed and the pellet briefly allowed to dry. Samples can then be passed for protein and/or RNA isolation.

'BioMAT'X, Facultad de Odontología, Universidad de Los Andes, Santiago de Chile.

${ }^{2}$ Plan de Mejoramiento Institucional (PMI) en Innovación I+D+i, Universidad de Los Andes, Santiago de Chile.

${ }^{3}$ Programa de Doctorado en BioMedicina, Facultad de Medicina, Universidad de Los Andes, Santiago de Chile.

${ }^{4}$ Centro de Investigación e Innovación Biomédica (CIIB), Facultad de Medicina, Universidad de Los Andes, Santiago de Chile. 
To permit clinical application, micro- and ultrafiltration techniques, have been recently introduced; allowing the rapid isolation of exosomes (urinary). Other methodologies also exist. In our experience at BioMAT'X, exosomes, (serum and salivary) are simply isolated following a precipitation protocol using the readilyavailable and efficient ExoQuickTM kit (System Biosciences, Mountain View, CA, USA). Amongst others, we do recommend this technique for exosomal miRNA profiling and exosomal RNA analysis (no conflict of interest exists). While it allows the use of small bio-fluid samples, with simplicity and rapidity, pellets will contain larger-sized exosomes. It is noteworthy also that, when compared to ultra-centrifugation, resulting samples from precipitation, will contain more non-exosomal-related proteins, microvesicles and membranous debris (biological impurities). Hence, pros and cons of each method should be taken into consideration, and selected per planned aims, objectives and requirements, for the research and/or use.

Quick Comments on Exosomes in Oral Cancer available studies are enormously limited. It seems thus far, that oral cancer-derived exosomes (salivary), when compared to those from healthy patients, are larger (20$400 \mathrm{~nm}$ ) in size, irregular in morphology (appear under Atomic Force Microscopy as circular or bulging vesicles) and are presented with elongated extra-vesicular nanofilaments, of un-known origin or function.

While both exosomes share similar protein markers (exosomal surface), variance in concentrations exist; higher expression of CD63 molecules in cancerous exosomes. Conversely, others reported decreases in CD9 and CD89 concentrations (via ELISA; insignificant differences). Recently, exosomes isolated from patients diagnosed with oral squamous cell carcinoma or OSCC, demonstrated an average size of 50-200 nm, using Scanning Electron Microscopy. Further, higher concentration levels of CD63 and CD81 molecules were detected via Western Blotting. Likewise, concentration levels of miR-21 were found to be higher than in healthy donors, interestingly. It is safe to say, again, nonetheless, that OSCC-related exosome literature is still sparse, as more focus or prioritization is given to characterizing and understanding human-derived salivary exosomes as novel nanobiomarkers for application in clinical diagnostics and therapeutics. In our BioMAT'X, ongoing efforts aim to explore potential use of exosomal concentration levels of CD63 and that of miR21 in circulating exosomes, for example, as an early indicator(s) for oral cancer onset, a measure for tumour aggressiveness (via studying oral pre-malignant lesions) and a biomarker for the diagnosis of oral cancer(s).

KEY WORDS: Exosomes; salivary diagnostics; salivary biomarker; oral fluid diagnostics; oral squamous cell carcinoma; oral cancer; salivaomics; proteomics; vesicles; nanosize.

Ziyad S. Haidar is a Full Professor of Biomaterials and Tissue Engineering and Scientific Director, Facultad de Odontología, Universidad de los Andes in Santiago de Chile. Concurrently, Prof. Dr. Haidar is the Founder and Head of the Biomaterials, Pharmaceutical Delivery and CranioMaxillo-Facial Tissue Engineering Laboratory (BioMAT'X Chile), Centro de Investigación e Innovación Biomédica (CIIB) and Faculty member in the Doctoral Program (BioMedicine) at the Facultad de Medicina, Universidad de los Andes in Santiago de Chile. Haidar is an oral and maxillofacial surgeon with a PhD in biomaterials, nanotechnology and tissue engineering from McGill University, Montréal, Canada; and post-doctoral training at the Montréal Shriners Hospital (Orthopedic Surgery), McGill University Health Center, Montréal, Canada. Before moving to Chile, he served as Associate Professor of Bioceramics and the Chair of Excellence in BioEngineering at the Université de Limoges, Limoges, France and was an Assistant Professor at the Department of Pharmaceutics and Pharmaceutical Chemistry (cross-appointment with Department of BioEngineering), University of Utah, Salt Lake City, UT, USA. Haidar served between 2010 and 2012 as Adjunct Professor of Head and Neck Surgery and the Scientific Director of the Research Center at Inha University Hospital, Seoul, South Korea. He has won several prestigious awards from the International Bone and Mineral Society, Society for Biomaterials, Canadian Biomaterial Society as well as the Canadian/Lebanese Societies of Plastic Surgeons, to name a few. He is an international speaker with $>100$ publications, conference proceedings, text-books and patents and is an editorial board member of several national and international scientific journals and periodicals.

Correspondance author:

Prof. Dr. Ziyad S. Haidar. DDS, Implantologist (Cert Implantol), Oral and Maxillofacial Surgeon (MSc), MBA, PhD. Professor and Scientific Director, Faculty of Dentistry, Universidad de Los Andes, Santiago de Chile. Founder and Head of BioMAT'X, Biomedical Research and Innovation Center (CIIB), PMI I+D+i, Department for Research, Development and Innovation, Universidad de Los Andes, Mons. Álvaro del Portillo 12.455 - Las Condes, Santiago, Chile. Telephone: +56226181372 Fax: +56 222149468

E-mail: zhaidar@uandes.cl

Recibido : 10-07-2017

Aceptado: 05-09-2017 